\title{
Pulsations and metallicity of the pre-main sequence eclipsing spectroscopic binary RS Cha ${ }^{\star}$
}

\author{
E. Alecian ${ }^{1}$, C. Catala ${ }^{1}$, C. Van't Veer-Menneret ${ }^{2}$, M.-J. Goupil ${ }^{1}$, and L. Balona ${ }^{3}$ \\ 1 Observatoire de Paris, LESIA, 5 place Jules Janssen, 92195 Meudon Principal Cedex, France \\ e-mail: evelyne.alecian@obspm.fr \\ 2 Observatoire de Paris, GEPI, 5 place Jules Janssen, 92195 Meudon Principal Cedex, France \\ 3 South African Astronomical Observatory, PO Box 9, Observatory 7935, Cape, South Africa
}

Received 26 August 2004 / Accepted 20 May 200

\section{ABSTRACT}

We present new spectroscopic observations of the pre-main sequence eclipsing spectroscopic binary RS Cha. A sample of 174 spectra were obtained with the GIRAFFE spectrograph at the SAAO at 32000 resolution. The radial velocity curves derived from these spectra were combined with previous observations spanning a period of about 30 years to correct the ephemeris of the system, and the result indicates that the orbital period is not constant.

Residuals of the binary radial velocity curve for both components with amplitudes up to a few $\mathrm{km} \mathrm{s}^{-1}$ and periods on the order of $1 \mathrm{~h}$ are clearly seen in our data, which we interpret as the signatures of delta-Scuti type pulsations.

We revisited the masses of both components and determined the surface metallicity $Z$ of both components of the RS Cha system by fitting synthetic spectra to observed spectra in a set of selected spectral regions. The synthetic spectra are calculated with the SYNTH code using stellar atmosphere models computed with the Kurucz ATLAS 9 code, along with a list of lines obtained from the VALD database. A selection of the best spectra and the most relevant spectral regions allowed us to determine $Z=0.028 \pm 0.005$. We also derived new values of $v \sin i$ : $64 \pm 6 \mathrm{~km} \mathrm{~s}^{-1}$ and $70 \pm 6 \mathrm{~km} \mathrm{~s}^{-1}$ for the primary and the secondary star, respectively. Finally, we observationally confirm that the RS Cha system is a synchronized and circularized system.

Key words. stars: abundances - stars: binaries: eclipsing - stars: binaries: spectroscopic - stars: pre-main-sequence stars: rotation - instrumentation: spectrographs

\section{Introduction}

Precise and reliable knowledge of masses, radii, temperatures, and metallicities of stars is fundamental for testing stellar evolutionary models, especially the pre-main sequence (PMS) phase. In the particular case of a double-lined eclipsing binary, masses and radii of the two components are known with a good accuracy. Hence valuable constraints for validating the physical input in evolutionary codes for the PMS phase will be available if one can determine effective temperatures and metallicities of both components of at least one PMS eclipsing binary system.

The bright A-type star RS Chamaeleontis has often been studied since its discovery as an eclipsing binary by Strohmeier (1964). Early spectroscopic and photometric studies of this star led to the determination of the orbit (Wild \& Lagerweij 1967; Chambliss 1967; Jones 1969). The photometric light curves of Wild \& Lagerweij (1967) and Chambliss (1967) were in good agreement and, in particular, both papers quote a luminosity ratio of about 2. However, Jorgensen (1975) re-examined the light curves and found equally bright components for this

* Based on observations collected at the South African Astronomical Observatory. system. Andersen (1975, hereafter A75) confirmed this new result with spectroscopic observations, then determined all physical parameters of both components and recalculated the orbital parameters. The age of RS Cha, member of the eta Cha cluster, has recently been estimated to 8 Myr by Mamajek et al. (2000) and to $6_{-1}^{+2}$ Myr by Luhman \& Steeghs (2004).

Observations of $u v b y$ light curves were analysed by Clausen \& Nordström (1978), who found photometric elements by comparing the eclipsing binary model developed by Wood (1971) with the observed photometric light curves Clausen \& Nordström (1980; hereafter CN80). The mass and radius $(M, R)$ of the two components were calculated with good accuracy and first values of effective temperatures $T_{\text {eff }}$ were determined using $(b-y), c_{1}$ values and the calibrations of $u v b y$ photometry by Breger (1974) and Relyea \& Kurucz (1978). Finally a more recent study (Ribas et al. 2000) of RS Cha based on the $u v b y \beta$ photometry led to a more accurate $(\sim 1 \%)$ determination of $T_{\text {eff. }}$.

As a result of all these efforts $M, R, T_{\text {eff }}$ are welldetermined for both components of RS Cha, but the metallicity of RS Cha was determined under the hypothesis that it is a post-MS star and using theoretical stellar evolution models. 
Jones (1969) indeed suggested that the stars are post-main sequence. A75, comparing the locus of RS Cha in the colourradius diagram and the theoretical stellar evolution models of Hejlesen (unpublished), found helium abundance of approximately $Y=0.25$ and placed the components about $1.5 \mathrm{mag}$ above the ZAMS at the end of the main sequence phase. $\mathrm{CN} 80$ found the same results with the same method and gave an $(Y, Z)=(0.28,0.02)$ chemical composition. Ribas et al. (2000) attempted to determine the chemical composition of RS Cha by comparing stellar evolutionary models and photometric data without reaching a satisfactory agreement.

All these results assumed that RS Cha is a post-MS star. However, Mamajek et al. (1999) recently reported X-ray emissions from RS Cha, which indicate a PMS status rather than a post-MS status as suggested before. Since then, no new determination of the metallicity of RS Cha has been performed.

A75 also reports small amplitude radial velocity variations on top of the binary radial velocity curve for both components of RS Cha, suggesting the possible presence of stellarpulsations. Later on, photometric observations by McInally \& Austin (1977) revealed short-term variations, possibly related to pulsations in at least one of the two components. These clues of stellar pulsation in RS Cha must be put in perspective with the recent prediction by Marconi \& Palla (1998) that PMS stars of intermediate mass, such as RS Cha, cross the $\delta$ Scuti instability strip for a significant fraction of their PMS phase, soon confirmed by the discovery of $\delta$ Scuti type pulsations in a dozen of them (see Catala 2003, for a review). The location of RS Cha in the HR diagram places this system right at the boundary of the instability strip predicted by Marconi \& Palla (1998) for the first three radial overtones, with one of the two components inside the strip and the other outside. In this framework, the search for pulsations in both components of this system is of great interest.

This paper presents a clear confirmation of the presence of $\delta$ Scuti-type pulsations in both components of RS Cha, as well as corrections of the binary system ephemeris and determination of the metallicity of both components based on a new set of spectroscopic observations. The spectroscopic observations and their reduction are presented in Sect. 2. Section 3 contains an analysis of the ephemeris of the binary system and shows evidence for pulsations in this binary system. Section 4 explains the fundamental parameters of the system used in our analysis. The method for deriving metallicity and the results are presented in Sect. 5. Finally the results are discussed in Sect. 6.

\section{Observations and reductions}

High resolution spectra of RS Cha were obtained at the South African Astronomical Observatory, using the GIRAFFE fibrefed echelle spectrograph attached to the 1.9 m Radcliffe telescope. This instrument is a copy of the MUSICOS spectrograph currently in use on the Bernard Lyot telescope at Pic du Midi Observatory, France (Baudrand \& Böhm 1992). The spectral resolution is about 32000 , corresponding to a width of 2 pixels of the $1024 \times 1024$ TEK CCD. A Th-Ar arc lamp was used for wavelength calibration. Arc spectra were taken at regular intervals to calibrate possible drifts. Flat-fielding was performed by taking the spectrum of a tungsten lamp and also by illuminating the CCD with uniform light through a diffusing screen. The blaze correction was determined by measuring the response across each order when the fibre was illuminated by a tungsten lamp. The wavelength range was 4400-6680 spread over 45 orders. Exposure times were around $10 \mathrm{~min}$, which yielded typical $\mathrm{S} / \mathrm{N}$ ratios between 40 and 75 per pixel, depending on the meteorological conditions and the airmass of the observations. A total of 174 spectra of RS Cha were recorded, 39 between Dec. 18 and 22, 2002, and 135 between Jan. 14 and 28, 2003 (cf. Table 1).

All spectroscopic data were reduced by one of us (LB), using the SPEC2 package described e.g. in Balona et al. (1996). In addition, special attention was paid to continuum normalization, since it has a serious impact on the measurement of equivalent widths and determination of line profiles and, hence, on the metallicity measurement. Because of the orbital motion of the binary system, the spectral lines have an excursion in the spectrum large enough that each wavelength bin in the spectrum is exposed only to continuum at least once during the monitoring. After proper smoothing in both time and wavelength spaces, we explored the full series of spectra and computed, for each wavelength bin, the 85 th percentile of the whole time series. This resulted in a first estimate of the continuum for each order of the spectrograph. A few remnants of the spectral lines still show up after this treatment, and we therefore automatically identified local maxima in this first estimate of the continuum by computing the 98 th percentile in the wavelength direction over 8 spectral bins per order, each one covering $1 / 8$ of the order. This procedure yielded a clean continuum for each order without any "eye" fitting. After this first continuum normalization, the spectra were subsequently re-normalized to the continuum by comparing each wavelength domain to corresponding synthetic spectra, as described in Sect. 5.5.

We then computed average photospheric profiles by applying the Least Square Determination (LSD) technique (Donati et al. 1997). This technique assumes that most spectral lines are broadened in the same way, and builds the average line profile by deconvolving the observed spectrum from a line mask including all lines computed in a synthetic spectrum of the star. We used a mask computed with a synthetic spectrum having $T_{\text {eff }}=7500 \mathrm{~K}$ and $\log g=4$, adequate for both components of the system. About 3000 spectral lines were used in the deconvolution. Typical S/N ratios of 500-600 per velocity bin of $3.5 \mathrm{~km} \mathrm{~s}^{-1}$ were obtained in the LSD average photospheric profiles.

\section{Radial velocity curves and stellar pulsations}

We measured the radial velocity curves of both components of the RS Cha system by fitting the LSD average line profiles with the sum of two functions modelling the line profiles of both components. Each one of these two functions is the convolution product of a Gaussian whose width is appropriate to the spectral resolution and a rotation function (Gray 1992). The free parameters of this fitting procedure are the centroids, depths, and $v \sin i$ values of both line components. An example of such a fit is shown in Fig. 1. This automatic fitting procedure is 
Table 1. List of observation periods. HJD = Heliocentric Julian Day.

\begin{tabular}{lllll}
\hline \hline Period & Date & HJD-2452 600.00 & Phase & Spectra number \\
\hline $\mathrm{a}$ & $18 / 12 / 02$ & {$[26.5717,26.6036]$} & {$[0.188,0.207]$} & {$[0,4]$} \\
$\mathrm{b}$ & $19 / 12 / 02$ & {$[27.5406,27.6039]$} & {$[0.769,0.806]$} & {$[5,13]$} \\
$\mathrm{c}$ & $20 / 12 / 02$ & {$[28.5500,28.5969]$} & {$[0.373,0.401]$} & {$[14,20]$} \\
$\mathrm{d}$ & $21 / 12 / 02$ & {$[29.5288,29.6105]$} & {$[0.959,0.008]$} & {$[21,31]$} \\
$\mathrm{e}$ & $22 / 12 / 02$ & {$[30.5525,30.6034]$} & {$[0.572,0.603]$} & {$[32,38]$} \\
$\mathrm{f}$ & $14 / 01 / 03$ & {$[54.4324,54.4716]$} & {$[0.873,0.896]$} & {$[39,44]$} \\
$\mathrm{g}$ & $15 / 01 / 03$ & 55.4959 & 0.509 & 45 \\
& $16 / 01 / 03$ & {$[55.5035,55.6143]$} & {$[0.514,0.580]$} & {$[46,60]$} \\
$\mathrm{h}$ & $17 / 01 / 03$ & {$[56.5047,56.6231]$} & {$[0.114,0.184]$} & {$[61,76]$} \\
$\mathrm{i}$ & $18 / 01 / 03$ & {$[57.5137,57.6245]$} & {$[0.718,0.784]$} & {$[77,91]$} \\
$\mathrm{j}$ & $19 / 01 / 03$ & {$[58.5118,58.6151]$} & {$[0.316,0.377]$} & {$[92,105]$} \\
$\mathrm{k}$ & $21 / 01 / 03$ & {$[60.5097,60.6230]$} & {$[0.512,0.580]$} & {$[106,120]$} \\
$\mathrm{l}$ & $22 / 01 / 03$ & {$[61.5680,61.6388]$} & {$[0.146,0.188]$} & {$[121,130]$} \\
$\mathrm{m}$ & $25 / 01 / 06$ & {$[64.5129,64.6237]$} & {$[0.909,0.976]$} & {$[131,145]$} \\
$\mathrm{n}$ & $26 / 01 / 03$ & {$[65.5264,65.6213]$} & {$[0.516,0.573]$} & {$[146,158]$} \\
$\mathrm{o}$ & $27 / 01 / 03$ & 66.5357 & 0.121 & 159 \\
$\mathrm{p}$ & $28 / 01 / 03$ & {$[67.5159,67.6192]$} & {$[0.708,0.769]$} & {$[160,173]$} \\
\hline
\end{tabular}

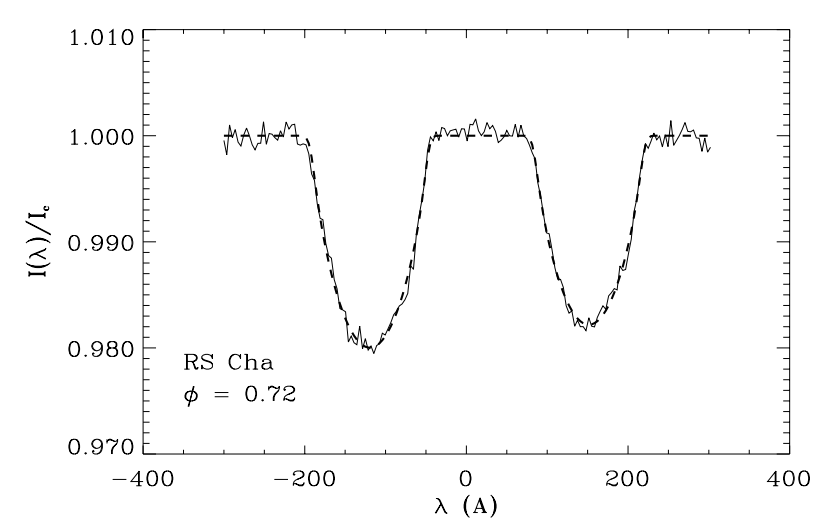

Fig. 1. Fit (dashed line) of the LSD profile (solid line) of one spectrum.

robust and enables us to measure the radial velocity and projected rotation velocity of both components. The typical error on radial velocities is only on the order of $0.3 \mathrm{~km} \mathrm{~s}^{-1}$, thanks to the high $\mathrm{S} / \mathrm{N}$ ratio of the LSD average photospheric profiles, while the error on $v \sin i$ is $6 \mathrm{~km} \mathrm{~s}^{-1}$.

We fitted the resulting radial velocity curves of both components of RS Cha phased with the ephemeris of CN80. We found a phase difference between our observations and the ephemeris of CN80 equal to 0.0449 , indicating that the ephemeris ought to be corrected. We used photometric and spectroscopic observations of RS Cha available in the literature (Schoeffel \& Kohler 1965; Chambliss 1967; Jones 1969; Andersen 1975; Clausen \& Nordström 1980; Mallama 1981) and data from Hipparcos and Tycho catalogues, combined with our own observations, to calculate the observed minus computed $(\mathrm{O}-\mathrm{C})$ timings of first and second conjunctions. A compilation of these timings is given in Table 2, as well as the $(\mathrm{O}-\mathrm{C})$ resulting from the ephemeris of $\mathrm{CN} 80$, chosen as a reference:

Min I : HJD $2442850.7688+n \times 1.669870$.

These results are also presented in Fig. 2.

If we disregard the times of first conjunctions by Schoeffel $\&$ Kohler (1965), which have a large dispersion, the (O-C) seem more or less constant until the mid 70's, then show a significant continuous increase up to now. The error bars for all measurements presented in Fig. 2 are sufficiently small to prove that the $(\mathrm{O}-\mathrm{C})$ will always vary with time in a non monotonic way, whatever the reference ephemeris chosen for the analysis. These variations seem too large to be due to the presence of a third body in the RS Cha system, but may be the signature of changes in the orbital period. Between 1975 and 2002, the observed changes in $(\mathrm{O}-\mathrm{C})$ would translate into a period variation rate $\dot{P} / P=7.7 \times 10^{-6} \mathrm{yr}^{-1}$. Further observations would be required to confirm and better characterize this orbital period variation and to understand its origin.

The radial velocity curve obtained from our data with a 0.0449 phase correction in order to match the CN80 ephemeris is shown in Fig. 3. Residuals to the binary radial velocity curve fit can be used to search for signatures of pulsations. These residuals are displayed in Fig. 4 and indeed present unambiguously regular variations, with variable amplitudes ranging from 1 to $3 \mathrm{~km} \mathrm{~s}^{-1}$ and periods on the order of $1 \mathrm{~h}$ for both components of the RS Cha system. Because our observations span less than $3 \mathrm{~h}$ per night, it proved impossible to derive reliable values for period(s) in these data. However, a possible frequency of 22.079 cycles/day is apparent for the most prominent variation of the primary component during nights $7,8,9$ with an amplitude of $0.89 \mathrm{~km} \mathrm{~s}^{-1}$. Although this frequency determination is not very reliable, it is very likely that the observed 
Table 2. Times of primary $(\mathrm{P})$ and secondary (S) minima and $\mathrm{O}-\mathrm{C}$.

\begin{tabular}{cccccc}
\hline \hline HJD-2 400 000 & Error & O-C & Error & Type & Reference \\
\hline 38380.537 & & 0.010 & & $\mathrm{P}$ & \\
38385.542 & & 0.006 & & $\mathrm{P}$ & \\
38406.412 & & 0.002 & & $\mathrm{~S}$ & Schoeffel \\
38442.353 & & 0.041 & & $\mathrm{P}$ & \& Kohler \\
38472.333 & & -0.037 & & $\mathrm{P}$ & $(1965)$ \\
38493.232 & & -0.011 & & $\mathrm{~S}$ & \\
38503.263 & & 0.001 & & $\mathrm{~S}$ & \\
\hline 38904.8655 & & -0.0005 & & $\mathrm{P}$ & \\
38930.7456 & & -0.0034 & & $\mathrm{~S}$ & \\
39136.1417 & & -0.0013 & & $\mathrm{~S}$ & Chambliss \\
39197.0925 & & -0.0007 & & $\mathrm{P}$ & \\
39207.9466 & & -0.0008 & & $\mathrm{~S}$ & \\
\hline 39222.1371 & 0.0009 & -0.0042 & 0.0034 & $\mathrm{P}$ & Jones (1969) \\
\hline 39232.9866 & & 0.0088 & & $\mathrm{~S}$ & \\
39233.8314 & & 0.0010 & & $\mathrm{P}$ & \\
39263.0508 & & -0.0023 & & $\mathrm{~S}$ & Chambliss \\
39283.9262 & & 0.0003 & & $\mathrm{P}$ & $(1967)$ \\
39284.7658 & & 0.0044 & & $\mathrm{~S}$ & \\
\hline 41720.2743 & 0.0009 & 0.0075 & 0.0019 & $\mathrm{P}$ & Andersen (1975) \\
\hline 42846.5951 & 0.0007 & 0.0010 & 0.0010 & $\mathrm{~S}$ & \\
42850.7692 & 0.0001 & 0.0004 & 0.0004 & $\mathrm{P}$ & \\
42851.6041 & 0.0001 & 0.0004 & 0.0004 & $\mathrm{~S}$ & Clausen \& \\
42855.7792 & 0.0001 & 0.0008 & 0.0004 & $\mathrm{P}$ & Nordström \\
42861.6230 & 0.0003 & 0.0000 & 0.0006 & $\mathrm{~S}$ & $(1980)$ \\
42870.8060 & 0.0001 & -0.0012 & 0.0004 & $\mathrm{P}$ & \\
42876.6507 & 0.0006 & -0.0011 & 0.0009 & $\mathrm{~S}$ & \\
\hline 44428.8047 & 0.0004 & 0.0088 & 0.0016 & $\mathrm{P}$ & Mallama (1981) \\
\hline 47867.0975 & 0.0009 & 0.0392 & 0.0042 & $\mathrm{P}$ & \multirow{2}{*}{ Hipparcos } \\
\hline 52626.2627 & 0.0006 & 0.0749 & 0.0068 & $\mathrm{P}$ & this paper \\
\hline
\end{tabular}

variations are due to $\delta$ Scuti type pulsations. These results confirm the conclusions of McInally \& Austin (1977) who found short-term photometric variations with 0.02 mag amplitudes.

The presence of $\delta$ Scuti pulsations in this well-constrained eclipsing binary system is extremely promising for future perspectives in asteroseismology. Further monitoring in high resolution spectroscopy with a much better coverage than we have here is highly needed in the near future. We finally searched for signatures of high degree modes, as line profile distortions. We did not see any profile distortion, down to a level of a few $10^{-3}$.

\section{Fundamental parameters of RS Cha}

The double-lined eclipsing binary RS Cha was the subject of several studies in the past, so that various values for the masses, radii, and effective temperatures of both components are available in the literature. Radii of both components were determined in the 70's with good accuracy. CN80 used Strömgren

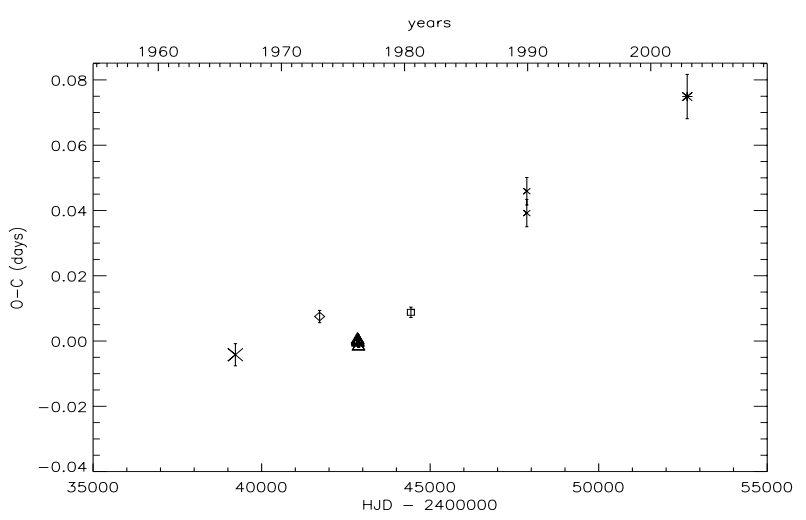

Fig. 2. (O-C) timings of the first conjunction over 30 years. Large cross: Jones (1969), diamond: Andersen (1975); triangles: Clausen \& Nordström (1980); open square: Mallama (1981); small crosses: Hipparcos; asteriks: this paper. Data of Schoeffel \& Kohler (1965) and Chambliss (1967) are not accurate enough to plot them.

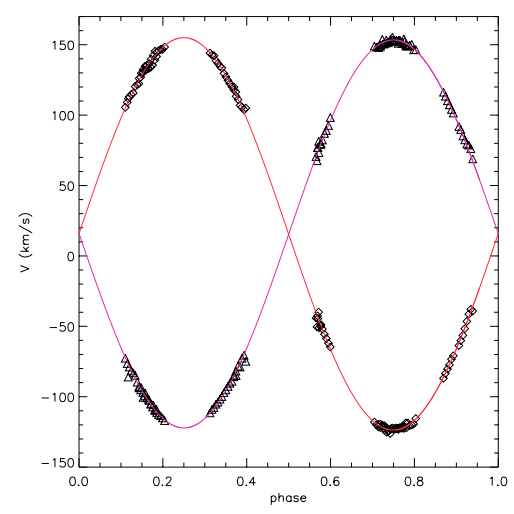

Fig. 3. Radial velocity curves of both components of RS Cha. Triangles: primary star; diamonds: secondary star.

four-colour $u v b y$ observations and the light curve simulation programme WINK described by Wood (1971) to determine these radii. They also determined the inclination of the orbit to high accuracy and confirmed the orbit is circular.

Unlike masses and radii, effective temperatures and luminosities of eclipsing binaries cannot be determined easily. Most authors (Wild \& Lagerweij 1967; Chambliss 1967; Jorgensen 1975 ) used broad band photometry to evaluate $T_{\text {eff }}$ for both primary and secondary components, but more reliable results can be obtained from intermediate-band, such as Strömgren photometry. CN80 used Strömgren $u v b y$ indices to calculate $T_{\text {eff }}$ for RS Cha with the calibration grids of Relyea \& Kurucz (1978) based on Kurucz (1979) stellar atmosphere models. More recently, Ribas et al. (2000) used Strömgren $u v b y \beta$ photometry of RS Cha and new improved photometric grids from Napiwotzki et al. (1993), based on Kurucz ATLAS 9 models, to determine effective temperatures of both components with an accuracy of $1 \%$. In this paper we adopt the effective temperatures of Ribas et al. (2000).

We redetermined the radial velocity amplitudes $K_{\mathrm{P}}$ and $K_{\mathrm{S}}$, using our data. The residual of our fit to the radial velocity curves has an rms of $1.9 \mathrm{~km} \mathrm{~s}^{-1}$, which is dominated by the presence of the pulsations described in Sect. 3. The accuracy of the determination of $K_{\mathrm{P}}$ and $K_{\mathrm{S}}$ is therefore limited by these 


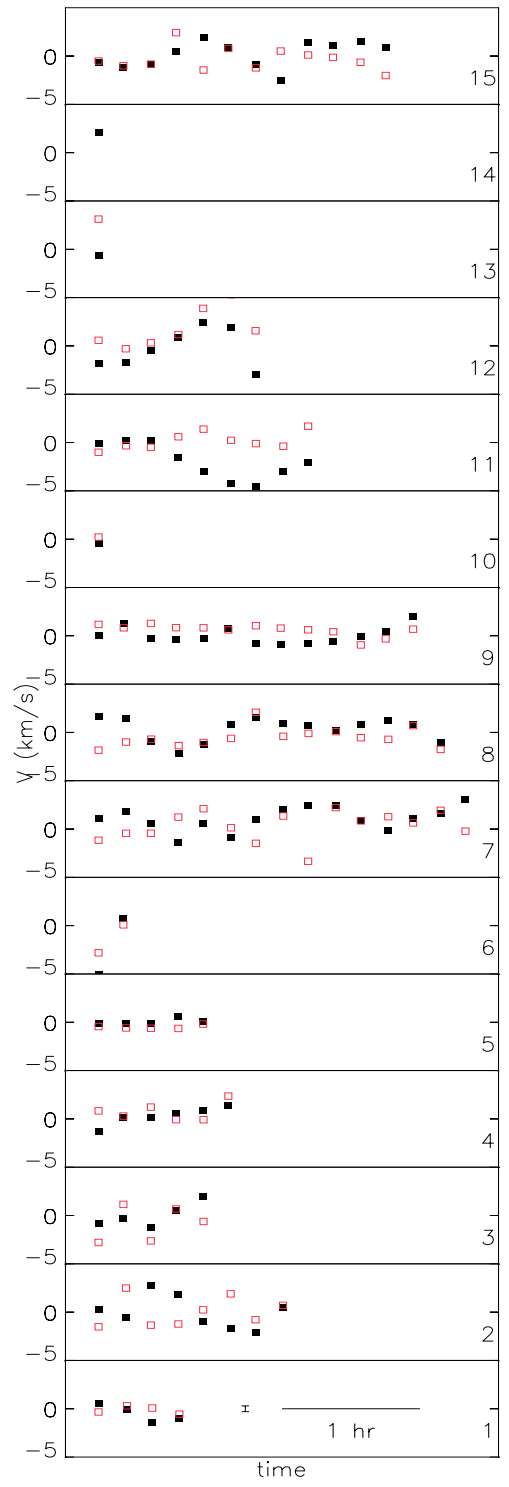

Fig. 4. Residuals of the binary radial velocity curves fitted to our data. Each night is presented separately, as labelled on the lower right of each panel. The typical error bar of $0.3 \mathrm{~km} \mathrm{~s}^{-1}$ is shown in the middle of the lower panel. Filled (resp. open) symbols belong to the primary (resp. secondary) component.

pulsations. However, because we have obtained a much larger number of data points than $\mathrm{A} 75$, our new value of $K_{\mathrm{P}}$ and $K_{\mathrm{S}}$ is less sensitive to the pulsations than that of A75 and is, therefore, likely to be more accurate. As a matter of fact, our results are significantly different from those of A75. We, therefore, redetermined the masses of both components of RS Cha, using our values for $K_{\mathrm{P}}$ and $K_{\mathrm{S}}$, and the orbital period and inclination from $\mathrm{CN80}$, as well as the projected semi-major axis $a \sin i$, and consequently the radii of both components. Our results are presented in Table 3.

All adopted fundamental parameters of RS Cha are given in Table 3. In the following, the subscript "P" refers to the component eclipsed at primary minimum and the subscript " $\mathrm{S}$ " to the secondary component.
Table 3. Fundamental parameters of RS Cha. CN80: Clausen \& Nordström (1980); ROO: Ribas et al. (2000); $\gamma$ : systemic radial velocity.

\begin{tabular}{|c|c|c|c|}
\hline & $\mathrm{P}$ & $\mathrm{S}$ & References \\
\hline$K\left(\mathrm{~km} \mathrm{~s}^{-1}\right)$ & $137.8 \pm 0.2$ & $139.4 \pm 0.2$ & this paper \\
\hline$M / M_{\odot}$ & $1.89 \pm 0.01$ & $1.87 \pm 0.01$ & this paper \\
\hline$R / R_{\odot}$ & $2.15 \pm 0.06$ & $2.36 \pm 0.06$ & this paper \\
\hline $\log \left(T_{\text {eff }}\right)(\mathrm{K})$ & $3.883 \pm 0.010$ & $3.859 \pm 0.010$ & R00 \\
\hline $\begin{array}{l}\log (g) \\
\left(\mathrm{cm} \mathrm{s}^{-2}\right)\end{array}$ & $4.05 \pm 0.06$ & $3.96 \pm 0.06$ & $g=M G / R^{2}$ \\
\hline $\log L\left(L_{\odot}\right)$ & $1.15 \pm 0.09$ & $1.13 \pm 0.09$ & $L=4 \pi R^{2} \sigma T_{\mathrm{efl}}^{4}$ \\
\hline$a \sin i\left(R_{\odot}\right)$ & \multicolumn{2}{|c|}{$9.14 \pm 0.01$} & this paper \\
\hline$i\left(^{\circ}\right)$ & \multicolumn{2}{|c|}{$83.4 \pm 0.3$} & $\mathrm{CN} 80$ \\
\hline$\gamma\left(\mathrm{km} \mathrm{s}^{-1}\right)$ & \multicolumn{2}{|c|}{$15.7 \pm 0.2$} & this paper \\
\hline
\end{tabular}

\section{Metallicity determination}

\subsection{Selection of spectra}

As a first step in this analysis, we selected from our series the spectra offering well separated lines for the two components of the binary system. With the geometric parameters of RS Cha given in Table 3, and using the ephemeris given as Eq. (1), we identified spectra observed during eclipses, and discarded them from our analysis. Finally, spectra with a low $\mathrm{S} / \mathrm{N}$ ratio were also discarded.

\subsection{Selection of lines and spectral regions}

We selected a set of spectral lines for this analysis, using the following criteria:

- Avoid intricate blends: due to the large projected rotation velocity, most lines are blended in the spectrum of both components; our selection of lines minimizes these blends.

- Avoid low S/N ratios: we selected only lines strong enough and in sufficiently well exposed spectral regions to stay clearly out of the noise; for some of the lines, only a fraction of the spectra can be used, the others being too noisy.

- Choose spectral lines offering a variety of excitation potentials and ionization stages, in order to verify the relevance of the atmospheric models.

This severe selection resulted in a set of 6 spectral lines which are listed in Table 4. The average number of spectra finally selected for the analysis is only 25 out of the whole series of 174 spectra. The list of spectra used for each line is presented in Fig. 9. Some of the selected lines appear independently on adjacent orders of the spectra, so we used the line profiles on both orders independently.

\subsection{Equivalent width measurements}

We measured the true equivalent widths (Gray 1992) of selected lines in selected spectra, separating the contribution of both components of the binary system in the following way. 
Table 4. Equivalent widths $W_{\mathrm{P}}, W_{\mathrm{S}}$ of selected lines.

\begin{tabular}{lllll}
\hline \hline$\lambda(\AA)$ & Ion & Order & $W_{\lambda \mathrm{P}}(\mathrm{m} \AA)$ & $W_{\lambda \mathrm{S}}(\mathrm{m} \AA)$ \\
\hline \multirow{2}{*}{4957.6} & \multirow{2}{*}{ FeI } & 115 & $356 \pm 32$ & $421 \pm 26$ \\
& & 114 & $365 \pm 34$ & $415 \pm 16$ \\
5316.6 & FeII & 106 & $302 \pm 27$ & $322 \pm 31$ \\
& & 127 & $597 \pm 14$ & $487 \pm 27$ \\
4481.1 & \multirow{2}{*}{ MgII } & 126 & $526 \pm 79$ & $536 \pm 21$ \\
5227.2 & \multirow{2}{*}{ FeI } & 108 & $322 \pm 30$ & $331 \pm 27$ \\
4454.8 & \multirow{2}{*}{ CaI } & 128 & $533 \pm 95$ & $510 \pm 114$ \\
& & 127 & $462 \pm 107$ & $562 \pm 74$ \\
5284.1 & \multirow{2}{*}{ FeII } & 108 & $247 \pm 34$ & $287 \pm 23$ \\
& & 107 & $252 \pm 42$ & $304 \pm 17$ \\
\hline
\end{tabular}

We first fitted the sum of two convolution products of a Gaussian and a rotation profile to the observed profiles, in a similar way as the treatment of the LSD average profiles described in Sect. 3. We then integrated the profiles of the two fitted functions independently to determine the apparent equivalent widths of the line, at the wavelength $\lambda$, for each component $\left(W_{\lambda \mathrm{P}}^{\prime}\right.$ and $\left.W_{\lambda \mathrm{S}}^{\prime}\right)$, and for the whole binary $\left(W_{\lambda}\right)$. Finally, the true equivalent widths of the two binary components can be computed as

$$
\begin{aligned}
& W_{\lambda \mathrm{P}}=W_{\lambda \mathrm{P}}^{\prime}+\beta_{\lambda}\left(W_{\lambda}-W_{\lambda \mathrm{S}}^{\prime}\right) \\
& W_{\lambda \mathrm{S}}=W_{\lambda \mathrm{S}}^{\prime}+\frac{W_{\lambda}-W_{\lambda \mathrm{P}}^{\prime}}{\beta_{\lambda}}
\end{aligned}
$$

where $\beta_{\lambda}=L_{\lambda \mathrm{S}} / L_{\lambda \mathrm{P}}$ is the luminosity ratio of the two components, at the specific wavelength of interest, and $L_{\lambda}=$ $4 \pi R^{2} \frac{2 h c^{2} / \lambda^{5}}{\mathrm{e}^{h c / \lambda k T}-1}$.

This procedure allows us to measure the equivalent widths of both components outside eclipses, even at phases where the lines of both components are blended due to their large rotational velocities. Table 4 gives the resulting equivalent widths for the selected lines.

Although the spectral regions were carefully selected to minimize line blends, the high rotational velocities of both components does not permit an abundance analysis using the curve of growth technique. Indeed this method is based on measuring the equivalent width of numerous lines of one element and adjusting the observed to synthetic $\log \left(\frac{W}{\lambda}\right)$ vs. $\log (X)$ curves (where $X=\log (g f \lambda)-\theta \chi, g$ and $f$ are the statistic weight and the oscillator strength of the line transition, $\theta=\frac{5040}{T}$, and $\chi$ is the excitation potential of the lower level of the transition) (Gray 1992) by varying the element abundance $A$. In the case of RS Cha, a single line is almost always a blend of many components (Fig. 5). Moreover the composition of the blend does not remain constant, but varies with the orbital phase. Thus, with our observations we cannot regroup a set of lines of only one element. We therefore adopted a more direct fitting method, based on a comparison of observed spectra with synthetic ones, which is described below.

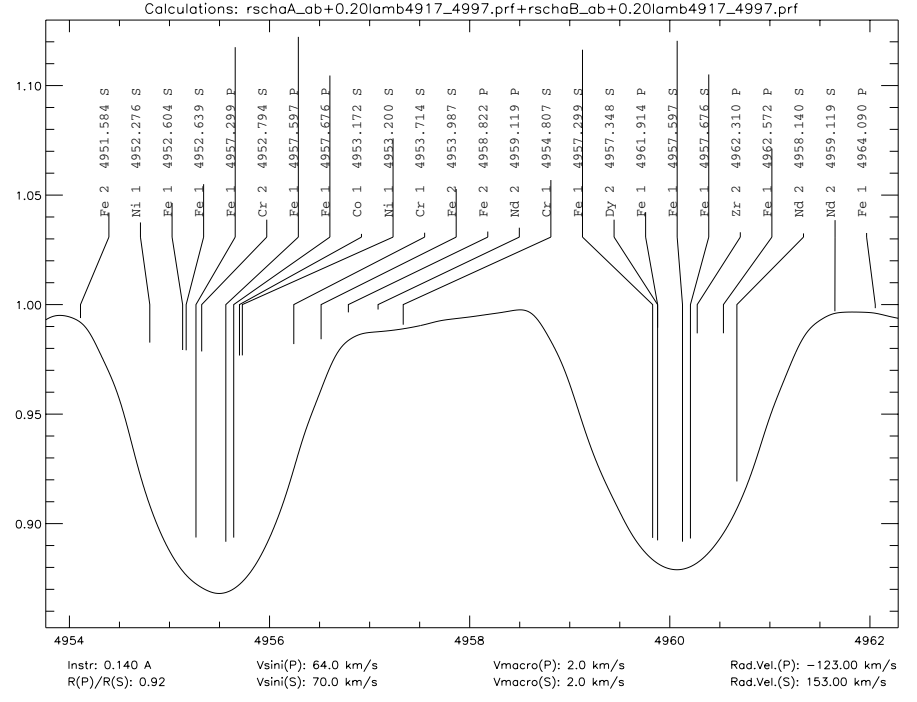

Fig. 5. Synthetic FeI $4957 \AA$ line computed with BINMAG1 (see text). The identification and the wavelength of each line are marked. $\mathrm{P}$ and $\mathrm{S}$ refer to the Primary or the Secondary star. The length of the bold stroke under the identification represents the relative intensity and the other stroke points to the center of the line.

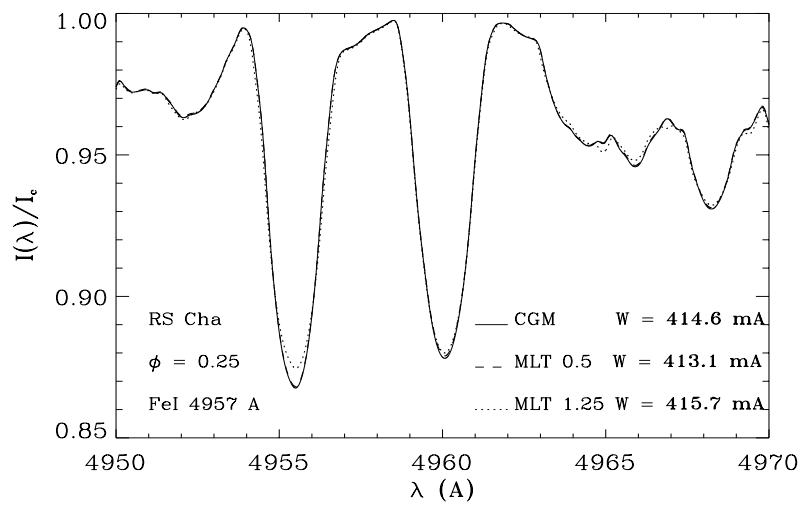

Fig. 6. Comparison of profile and equivalent width for three different convection treatments: CGM theory (solid line), $\alpha=0.5$ MLT (dashed line), $\alpha=1.25$ MLT (dotted line). The solid line and the dashed line are superimposed.

\subsection{Calculation of synthetic profiles}

\subsubsection{The choice of the convection treatment}

Synthetic spectra of each one of the two components were calculated with the SYNTH code of Piskunov (1992). This code needs a stellar atmosphere model and a list of lines as input. We used the Kurucz ATLAS 9 code to calculate stellar atmosphere models. We considered three different treatments of convection: the mixing length theory (MLT) with (i) $\alpha=0.5$ and (ii) $\alpha=1.25$, and (iii) the CGM convection model (Canuto et al. 1996). Figure 6 compares the profiles obtained in the region of the Fe I $4957 \AA$ line with these various treatments of convection, and shows that this choice does not have a large impact on the metallicity determination. The CGM theory and MLT $\alpha=0.5$ profiles of the FeI $4957 \AA$ line are exactly identical, while the MLT $\alpha=1.25$ profile is slightly different; but in all three cases the equivalent widths of the line are nearly 
Table 5. Model parameters used to calculate the synthetic spectrum of RS Cha. In addition, the projected rotation velocity $v \sin i$ and the heavy elements abundance $Z$ are free parameters, which are determined in the following sections. The hydrogen abundance $(X)$ and the helium abundance $(Y)$ have solar values in first approximation.

\begin{tabular}{lllll}
\hline \hline star & $\begin{array}{l}T_{\text {eff }} \\
(\mathrm{K})\end{array}$ & $\begin{array}{l}\log (g) \\
\left(\mathrm{cm} \mathrm{s}^{-2}\right)\end{array}$ & $\alpha$ & $\begin{array}{l}v_{\text {micro }} \\
\left(\mathrm{km} \mathrm{s}^{-1}\right)\end{array}$ \\
\hline Primary & 7640 & 4.05 & 0.5 & 2 \\
Secondary & 7230 & 3.95 & 0.5 & 2 \\
\hline
\end{tabular}

identical. Following Heiter et al. (2002), we used $\alpha=0.5$ in calculating synthetic spectra.

\subsubsection{The check of the $\log (g f)$ values}

The line list was obtained from the VALD database ${ }^{1}$ (Vienna Atomic Line Database), which provides all lines and their parameters in any chosen spectral interval for any chosen star. Among the listed parameters, the $\log (g f)$ values need to be thoroughly checked, since they are fundamental to this work. In order to verify them, we compared the equivalent widths of the studied lines in a well-calibrated high resolution solar spectrum (Kurucz et al. 1984) to a synthetic solar spectrum calculated with ATLAS9 and SYNTH, using the line list provided by the VALD database. We found that the VALD tabulated values for all studied lines were in good agreement.

\subsubsection{Calculation of synthetic spectra}

Using the SYNTH code, we then calculated synthetic spectra in spectral regions $80 \AA$ wide, centered on the chosen lines for this analysis, and independently for each one of the two components of the binary system. The final spectrum of the double-lined eclipsing binary was computed with the BINMAG1 program developed by Oleg Kochukhov (private communication). This code uses synthetic spectra as input calculated with the SYNTH code, and calculates the resulting spectrum of the binary, taking into account the radii and temperatures of both components, their radial, rotational, and microturbulent velocities, as well as the resolving power of the instrument. Instrumental and microturbulent broadening are taken as Gaussian, while rotational broadening is calculated following Gray (1992).

Finally, the combination of these three codes provides us with normalized synthetic spectra of the eclipsing binary RS Cha in a spectral interval centered on each line studied. Table 5 lists all model parameters used in this study.

\subsection{Comparison of observed spectra with synthetic profiles}

As explained in Sect. 5.3, the curve-of-growth method cannot be used to measure the metallicity of RS Cha, because of the large $v \sin i$ of both components. We therefore choose to fit the

\footnotetext{
${ }^{1}$ http://www.astro.univie.ac.at/vald/
}

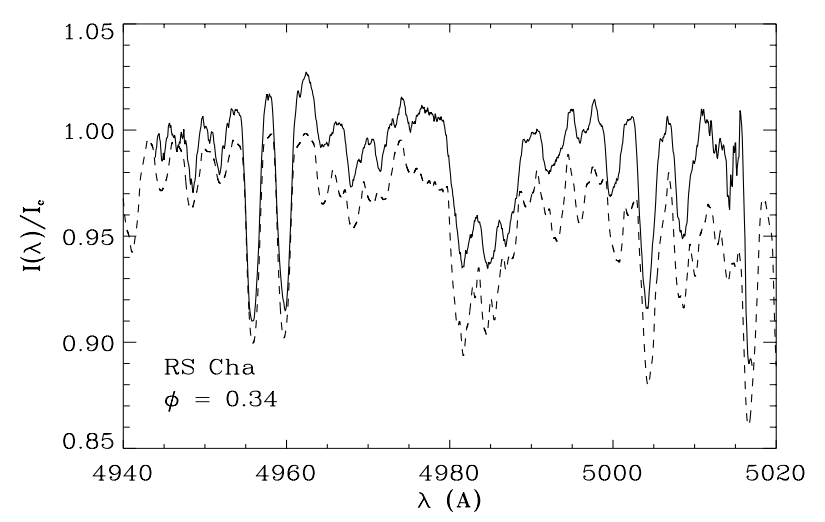

Fig. 7. Unnormalized observed (solid line) and synthetic (dashed line) spectra surronding the FeI $4957 \AA$ line. The synthetic spectrum was computed for $Z=0.030$.

spectral region around each line listed in Table 9 with synthetic spectra. The metallicity $Z$ is assumed identical in both components. Rotational velocities of the primary and secondary components $(v \sin i(P), v \sin i(S))$ are also varied. The metallicity $Z$ is defined as

$Z=\frac{\sum_{\text {metals }} \mu_{\mathrm{i}} N_{\mathrm{i}}}{\sum_{\text {metals }} \mu_{\mathrm{i}} N_{\mathrm{i}}+\mu_{\mathrm{He}} N_{\mathrm{He}}+\mu_{\mathrm{H}} N_{\mathrm{H}}}$

where $\mu_{\mathrm{i}}$ is the atomic weight (per atom) of the $i$ th element, $N_{\mathrm{i}}$ the number of atoms, and the sums are taken over all heavy elements, assuming solar relative abundances.

First we need to adjust the continua of the synthetic and observed spectra, which is a difficult task since the high rotational velocity does not leave very much continuum between the lines in the spectrum, as illustrated in Fig. 7. We therefore choose to normalize both the synthetic and observed spectra on either side of each line considered in this study, without attempting a better normalization on wider wavelength domains. After this renormalization, synthetic profiles are directly comparable to observed ones in a small wavelength region around each line.

The method basically consists in an eye-fitting of the synthetic spectrum, calculated as described in Sect. 5.4 to the observed spectrum, after the renormalization detailed above. We assume that both components of the binary have the same metallicity, and the same relative abundances of heavy elements, which is reasonable since both components were presumably initially formed in the same parental cloud. We further assumed that the relative abundances of heavy elements are identical to the solar ones. An example of a final fit to the observed FeI $4957 \AA$ line profile of one spectrum is shown in Fig. 8 on the left. We observe on the right that the synthetic profile does not fit the observed profile for an abundance outside the error bars.

We finally obtained, for each spectral region considered, a set of models matching the observed profiles of the various spectra in the series. All these models differ only by their values of $Z, v \sin i(P)$ and $v \sin i(S)$. For each spectral region, we then calculated the average value and dispersion of $Z$, $v \sin i(P)$ and $v \sin i(S)$ for all spectra in the series. Table 6 
Table 6. Different spectral regions and the $Z$ value which fits the associated spectrum in this region. $\chi_{\mathrm{ex}}$ is the excitation potential of the transition of the dominant ion.

\begin{tabular}{llllll}
\hline \hline Spectral region $(\AA)$ & Dominant ion & $\chi_{\mathrm{ex}}(\mathrm{eV})$ & Order & {$[\mathrm{Fe} / \mathrm{H}]$} & $Z$ \\
\hline$[4947.4,4967.4]$ & FeI & 2.808 & 115 & $0.18 \pm 0.04$ & $0.029 \pm 0.002$ \\
& & & 114 & $0.17 \pm 0.05$ & $0.029 \pm 0.003$ \\
{$[5306.7,5326.7]$} & FeII & 3.153 & 106 & $0.17 \pm 0.05$ & $0.029 \pm 0.003$ \\
{$[4471.1,4491.1]$} & MgII & 8.864 & 127 & $0.16 \pm 0.03$ & $0.028 \pm 0.002$ \\
& & & 126 & $0.17 \pm 0.04$ & $0.029 \pm 0.003$ \\
{$[5217.2,5237.2]$} & FeI & 1.557 & 108 & $0.17 \pm 0.05$ & $0.028 \pm 0.003$ \\
{$[4444.8,4464.8]$} & CaI & 1.899 & 128 & $0.17 \pm 0.05$ & $0.029 \pm 0.003$ \\
& & & 127 & $0.16 \pm 0.03$ & $0.028 \pm 0.002$ \\
{$[5274.1,5294.1]$} & FeII & 2.891 & 108 & $0.16 \pm 0.03$ & $0.028 \pm 0.002$ \\
& & & 107 & $0.15 \pm 0.04$ & $0.027 \pm 0.002$ \\
\hline
\end{tabular}
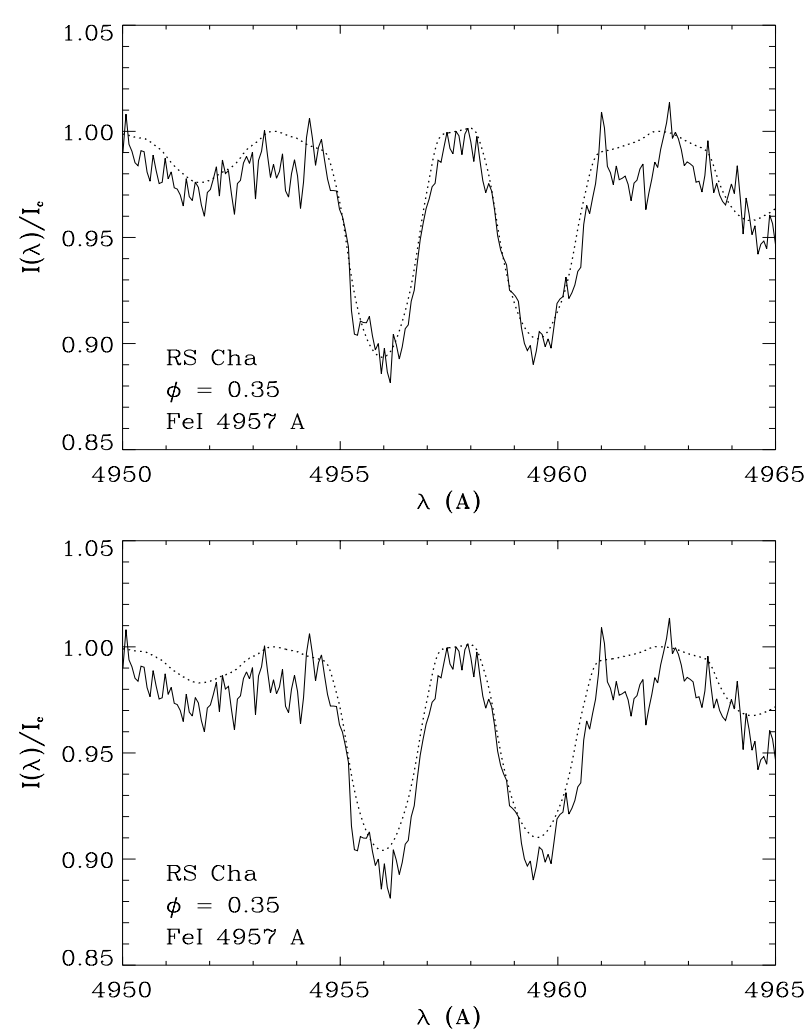

Fig. 8. Synthetic (dotted line) and observed FeI $4957 \AA$ line (solid line) computed with two different abundances: $Z=0.027$ above and $Z=0.012$ below.

summarizes the results of $[\mathrm{Fe} / \mathrm{H}]\left([\mathrm{Fe} / \mathrm{H}]=\log \left(\frac{N_{\mathrm{Fe}}}{N_{\mathrm{H}}}\right)_{\mathrm{RSCha}}-\right.$ $\left.\log \left(\frac{N_{\mathrm{Fe}}}{N_{\mathrm{H}}}\right)_{\odot}\right)$ and $Z$ for each spectral region. The tabulated $1 \sigma$ accuracies for $[\mathrm{Fe} / \mathrm{H}]$ in Table 6 are determined by the dispersion calculated as described above. The values of $v \sin i$ were found equal to $64 \pm 6 \mathrm{~km} \mathrm{~s}^{-1}$ and $70 \pm 6 \mathrm{~km} \mathrm{~s}^{-1}$ for the primary and the secondary components, respectively, for all studied lines.

As a final step, we estimated the best determination of the metallicity of RS Cha by averaging all values of Table 6 . We checked that the series of $[\mathrm{Fe} / \mathrm{H}]$ obtained with different lines are not correlated with one another. We therefore conclude that the final internal accuracy of $[\mathrm{Fe} / \mathrm{H}]$ when using all lines is improved by $\sqrt{N}$, where $N$ is the number of lines. However, the assumption that the relative abundances of heavy elements are identical to the solar ones is central to our method of measurement, and the expected dispersion about this ideal situation must be taken into account for a reliable estimate of the error bar on our determination of $Z$.

If we denote $N_{\mathrm{i}}$ as the number density of species $i$, and $N_{\text {ref }}$ the number density of a reference species (e.g. iron, but the final results do not depend on the choice of reference species), we can rewrite Eq. (4) for the metallicity $Z$ at first order as

$Z=\frac{\sum_{\text {metals }} \mu_{\mathrm{i}}\left(\frac{N_{\mathrm{i}}}{N_{\text {ref }}}\right)_{\odot} x_{\mathrm{i}}}{\sum_{\text {metals }} \mu_{\mathrm{i}} \frac{N_{\mathrm{i}}}{N_{\text {ref }}}+\mu_{\mathrm{He}} \frac{N_{\mathrm{He}}}{N_{\text {ref }}}+\mu_{\mathrm{H}} \frac{N_{\mathrm{H}}}{N_{\text {ref }}}}$

where $x_{\mathrm{i}}$ is the ratio of relative abundance of species $i$ with respect to the reference species to the same relative abundance in the sun

$x_{\mathrm{i}}=\frac{N_{\mathrm{i}} / N_{\text {ref }}}{\left(N_{\mathrm{i}} / N_{\text {ref }}\right)_{\odot}}$.

The dispersion on $\mathrm{Z}$ induced by the dispersion on the $x_{\mathrm{i}}$ 's is then:

$\mathrm{d} Z=\frac{\sum_{\text {metals }} \mu_{\mathrm{i}}\left(\frac{N_{\mathrm{i}}}{N_{\text {ref }}}\right)_{\odot} \mathrm{d} x_{\mathrm{i}}}{\sum_{\text {metals }} \mu_{\mathrm{i}} \frac{N_{\mathrm{i}}}{N_{\text {ref }}}+\mu_{\mathrm{He}} \frac{N_{\mathrm{He}}}{N_{\text {ref }}}+\mu_{\mathrm{H}} \frac{N_{\mathrm{H}}}{N_{\text {ref }}}}$

where $\mathrm{d} x_{\mathrm{i}}$ is the dispersion on $x_{\mathrm{i}}$.

We estimated the $\mathrm{d} x_{\mathrm{i}}$ 's empirically, using the abundances of a large sample of A and F stars, measured by Erspamer and North (2003). From their initial sample of 140 stars, we extracted a sub-sample of 16 stars, after discarding chemically peculiar stars, fast rotators $\left(v \sin i>50 \mathrm{~km} \mathrm{~s}^{-1}\right)$, and stars with poor abundance determinations. For each one of the 28 elements considered in Erspamer and North's analysis, we determined the dispersion $\mathrm{d} x_{\mathrm{i}}$, and used these dispersions to calculate the dispersion on $Z$ as given by Eq. (7). We checked that changing the reference species does not affect the dispersion on $Z$, as expected. The final results on $Z$ and its overall error bar including the impact of our assumption about relative abundances, discussed above, are presented in Table 7. We checked 

spectral region

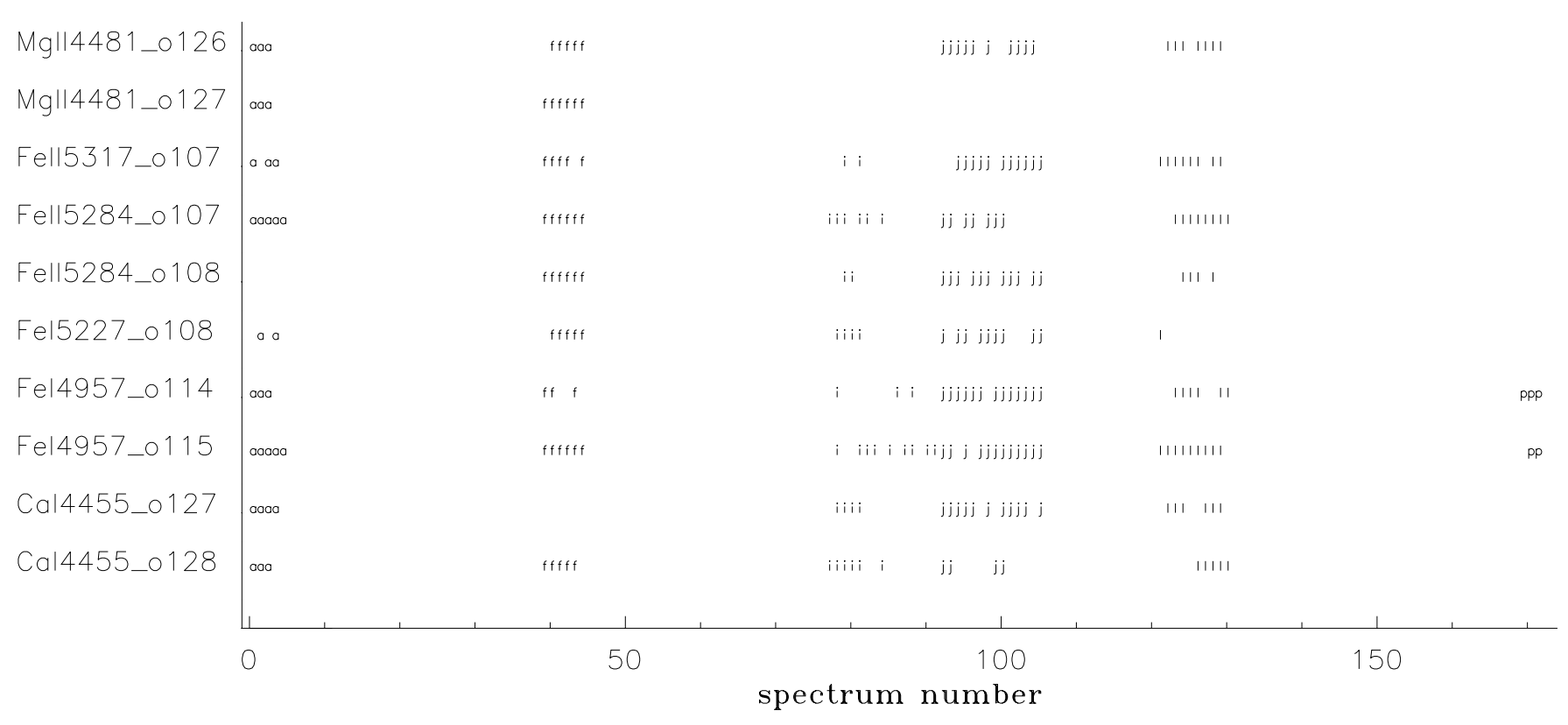

Fig. 9. Spectrum list chosen to study the spectral regions. The letters refer to the observation period explained in Table 1. The number besides the line name is the order number.

Table 7. Parameters of the final model representing RS Cha. We have taken solar abundances equal to: $X_{\odot}=0.707, Y_{\odot}=0.274, Z_{\odot}=0.019$. The error bar indicated for $[\mathrm{Fe} / \mathrm{H}]$ is internal.

\begin{tabular}{|c|c|c|c|c|c|c|c|}
\hline star & $\begin{array}{l}T_{\text {eff }} \\
(K)\end{array}$ & $\begin{array}{l}\log (g) \\
\left(\mathrm{cm} \mathrm{s}^{-2}\right)\end{array}$ & $\alpha$ & $\begin{array}{l}v_{\text {micro }} \\
\left(\mathrm{km} \mathrm{s}^{-1}\right)\end{array}$ & $\begin{array}{l}v \sin i \\
\left(\mathrm{~km} \mathrm{~s}^{-1}\right)\end{array}$ & {$[\mathrm{Fe} / \mathrm{H}]$} & $Z$ \\
\hline$P$ & 7640 & 4.05 & 0.52 & & $64 \pm 6$ & \multirow{2}{*}{$0.17 \pm 0.01$} & \multirow{2}{*}{$0.028 \pm 0.004$} \\
\hline$S$ & 7230 & 3.95 & 0.52 & 2 & $70 \pm 6$ & & \\
\hline
\end{tabular}

that varying $\log g$ up to \pm 0.07 dex and $\log T_{\text {eff }}$ up to \pm 0.01 dex, the resulting values of $Z$ remain within the error bars indicated in Table 7.

\section{Discussion}

The results presented in previous sections lead to several conclusions. First of all, we find a ratio of $v \sin i$ for both components of the binary equal to the ratio of their radii, which demonstrates that the system is synchronized. It seems to be in good agreement with the prediction of tidal evolution, since:

- the RS Cha system is known to be circularized since CN80 calculated the eccentricity $e \sin \omega=-0.013 \pm 0.004$.

- the tidal friction in such a system results in a synchronization time shorter than the circularization time (Zahn 1977).

- Mayer \& Hanna (1991) calculated a circularization time of 5 Myr for RS Cha, shorter than the age of RS Cha (Mamajek et al. 2000; Luhman \& Steeghs 2004).

The values of $Z$ found from different species and ionization stages are identical within the error bars. In particular we used in our study pairs of lines from the same species in the same ionization stage (but of different excitation potentials, such as Fe I $4957 \AA$ and $5227 \AA$ ), as well as lines from successive ionization stages of the same species such as [Fe I,Fe II]. This demonstrates that the choice of effective temperatures and gravities for both components, as given in Table 3, is correct. Moreover, we checked that varying $T_{\text {eff }}$ and $\log (g)$ within their error bars gives consistent values of $Z$ within the error bars interval for various lines. We therefore confirm the adopted values of $T_{\text {eff }}$ and $\log (g)$ taken from the literature.

It is important to note that we derived the same values of $Z$ from different elements: [Fe, $\mathrm{Mg}, \mathrm{Ca}$ ], which confirms that relative abundances of metals are identical to the solar ones, as assumed.

We have assumed in our analysis that the RS Cha components are not Am stars. This is justified, as Michaud et al. (1983) have shown that the disappearance of the HeII convection zone is required to lead to the Am phenomenon. The HeII convection zone can disappear if helium is sufficiently depleted by gravitational settling. This process is very slow, and is efficient only for slowly rotating stars. The components of RS Cha are both much too young and rotating too rapidly for this process to take place.

The surface metallicity determined in this paper can now be used in conjunction with the masses, radii, temperatures, and luminosities of both components, taking into account that they are coeval and assuming that the surface metallicity corresponds to the metallicity of the whole star to constrain the models of PMS evolution (in progress).

Acknowledgements. We are very grateful to O. Kochukhov, who provided his BINMAG1 code. We wish to thank Caroline Barban for her help calculating stellar atmosphere models. We are grateful to David Katz for his script allowing us to easily calculate Kurucz stellar atmosphere models. We thank R. L. Kurucz and N. E. Piskunov for authorizing the use of their ATLAS9 code and SYNTH code, respectively. 
This research made use of the VALD database operated by the Institut fuer Astronomie in Vienna, Austria. We wish to thank Jean-Paul Zahn and George Alecian for their advice on the circularization and synchronization times of a double system, and about the Am stars, respectively. Finally we thank the referee for his judicious comments, which led to major improvements in the paper.

\section{References}

Abt, H. A. 1967, in Magnetic and Related Stars, 173

Abt, H. A., \& Bidelman, W. P. 1969, ApJ, 158, 1091

Andersen, J. 1975, A\&A, 44, 445

Andersen, J. 1991, A\&A Rev., 3, 91

Balona, L. A., Böhm, T., Foing, B. H., et al. 1996, MNRAS, 281, 1315

Baudrand, J., \& Böhm, T. 1992, A\&A, 259, 711

Breger, M. 1974, ApJ, 192, 77

Canuto, V. M., Goldman, I., \& Mazzitelli, I. 1996, ApJ, 473, 550

Castelli, F. 1991, A\&A, 251, 106

Catala, C. 2003, Ap\&SS, 284, 53

Chambliss, C. R. 1967, AJ, 72, 518

Clausen, J. V., \& Nordström, B. 1978, A\&AS, 33, 87

Clausen, J. V., \& Nordström, B. 1980, A\&A, 83, 339

Conti, P. S. 1970, PASP, 82, 781

Donati, J.-F., Semel, M., Carter, B. D., Rees, D. E., \& Collier Cameron, A. 1997, MNRAS, 291, 658

Erspamer, D., \& North, P. 2002, A\&A, 383, 227

Erspamer, D., \& North, P. 2003, A\&A, 398, 1121

ESA 1997, The Hipparcos and Tycho Catalogues

Gray, D. F. 1992, The observation and analysis of stellar photospheres (Cambridge Astrophysics Series, Cambridge: Cambridge University Press, 2nd ed., ISBN 0521403200.)

Heiter, U., Kupka, F., Van’t Veer-Menneret, C., et al. 2002, A\&A, 392, 619

Jones, D. H. P. 1969, Mon. Notes Astron. Soc. S. Afr., 28, 5

Jordi, C., Ribas, I., Torra, J., \& Gimenez, A. 1997, A\&A, 326, 1044

Jorgensen, H. E. 1975, A\&A, 44, 459
Kallrath, J., \& Milone, E. F. 1999, Eclipsing binary stars: modeling and analysis

Kurucz, R. 1993, ATLAS9 Stellar Atmosphere Programs and $2 \mathrm{~km} \mathrm{~s}^{-1}$ grid. Cambridge, Mass.: Smithsonian Astrophysical Observatory

Kurucz, R. L. 1979, ApJS, 40, 1

Kurucz, R. L., Furenlid, I., \& Brault, J. 1984, Solar flux atlas from 296 to 1300 NM (National Solar Observatory Atlas, Sunspot, New Mexico: National Solar Observatory)

Lagerweij, H. C. 1967, Mon. Notes Astron. Soc. S. Afr., 26, 53

Luhman, K. L., \& Steeghs, D. 2004, ApJ, 609, 917

Mallama, A. D. 1981, PASP, 93, 774

Mamajek, E. E., Lawson, W. A., \& Feigelson, E. D. 1999, ApJ, 516, L77

Mamajek, E. E., Lawson, W. A., \& Feigelson, E. D. 2000, ApJ, 544, 356

Marconi, M., \& Palla, F. 1998, ApJ, 507, L141

Mayer, P., \& Hanna, M. A.-M. 1991, Bull. astr. Inst. Czechosl., 42, 98

McInally, C. J., \& Austin, R. D. 1977, Informational Bulletin on Variable Stars, 1334, 1

Michaud, G., Tarasick, D., Charland, Y., \& Pelletier, C. 1983, ApJ, 269, 239

Moon, T. T., \& Dworetsky, M. M. 1985, MNRAS, 217, 305

Napiwotzki, R., Schoenberner, D., \& Wenske, V. 1993, A\&A, 268, 653

Piskunov, N. E. 1992, in Stellar Magnetism, 92

Relyea, L. J., \& Kurucz, R. L. 1978, ApJS, 37, 45

Ribas, I., Jordi, C., Torra, J., \& Giménez, Á. 2000, MNRAS, 313, 99

Schoeffel, E., \& Kohler, U. 1965, Informational Bulletin on Variable Stars, 77,1

Smith, M. 1971, BAAS, 3, 11

Smith, M. A. 1972, ApJ, 175, 765

Strömgren, B. 1966, ARA\&A, 4, 433

Strohmeier, W. 1964, Informational Bulletin on Variable Stars, 55, 1

Wild, P. A. T., \& Lagerweij, H. C. 1967, Mon. Notes Astron. Soc. S. Afr., 26, 144

Wood, D. B. 1971, AJ, 76, 701

Zahn, J.-P. 1977, A\&A, 57, 383 